\title{
A Study on the Analysis of Particle Size Distribution for Bimodal Model Nanoparticles by Electron Microscopy
}

Christoph Hörenz ${ }^{1}$, Olivier Tache ${ }^{2}$, Dorota Bartczack ${ }^{3}$, Susana Nunez ${ }^{3}$, Isabel Abad Alvaro ${ }^{3}$, Heidi Goenaga-Infante $^{3}$ and Vasile-Dan Hodoroaba ${ }^{1}$

${ }^{1}$ Federal Institute for Materials Research and Testing (BAM), Berlin, Berlin, Germany, ${ }^{2}$ Université ParisSaclay, CEA, CNRS, NIMBE, Gif-sur-Yvette, Ile-de-France, France, ${ }^{3}$ National Measurement Laboratory, LGC, Teddington, England, United Kingdom

Measurement of nanoparticle size distribution (PSD) involves the accurate measurement of the size of the nanoparticles (NPs), i.e. abscissa of the PSD, as well as the accurate counting of the nanoparticles, i.e. the ordinate of the PSD [1]. The 'pure' size measurement of spherical NPs by electron microscopy can be carried out reliably on a routine basis, for both monodisperse and polydisperse samples. If the NPs can be prepared on a substrate such that the particles are separated (do not agglomerate), the way for reliable automated analysis (including measurement) is open. This is mostly the case for 'friendly' particulate materials such as (nearly) spherical silica, gold or polystyrene NPs [2,3]. The task becomes challenging when NPs of more complex shapes are involved [1,4], respectively when the absolute particle number concentration of multimodal size distributions must be determined by electron microscopy $[1,5,6]$. The latter case can be managed to a more or lesser extent with the electron microscopy by considering additional instrumental approaches like micro-liquid dispenser and gravimetry [5] or electrospray ionization [6].

The present study addresses the capability of measurement of relative particle number concentration by scanning electron microscopy for model bimodal silica and gold samples prepared in the frame of the European research project "nPSize - Improved traceability chain of nanoparticle size measurements" [7] as candidate reference nanoparticles.

The following samples have been investigated: i) bimodal, spherical silica NPs of 30 and $60 \mathrm{~nm}$ nominal size with nominal intensity ratios of 1:1 and 9:1, [8] ii) bimodal, (close-to) spherical gold NPs of $30 \mathrm{~nm}$ and $60 \mathrm{~nm}$ nominal size with nominal intensity ratios of 1:1 and 10:1. [9] The NPs have been prepared from the liquid suspension by drop-casting on conventional carbon-coated TEM grids.

Figure 1 shows representative SEM and STEM-in-SEM images of selected NP materials under investigation. While the SEM micrographs (Figure 1, left) do not allow their accurate analysis due to partially agglomerated NPs and contamination of organic material, the STEM-in-SEM micrographs (Figure 1, center) reveal distinct particle boundaries due to superior material contrast rendering more accurate image analysis, e.g. via ImageJ. As indicated in the associated particle size distributions (Figure 1, right), the nominal and measured relative particle concentration are almost identical. However, successful particle analysis is strongly dependent on a proper sample preparation, the number of counted particles, the physical resolution of the microscope as well as the digital resolution (pixel size) setting of the software used for image acquisition. The next step of reliable measurements of absolute NP concentrations can be reached only with a perfectly homogeneous dispersion of the NPs from a suspension droplet of known volume on a substrate - ideally a monolayer of non-overlapping particles.

In conclusion, the study confirms that the size measurement of bimodal spherical NPs by electron microscopy is relatively robust, rather independent of the measurement conditions. In contrast, the measurement of the absolute NP concentration is not possible due to the heterogeneity of particle 
dispersion on the substrate. Determination of the relative NP concentration is indeed possible, however, under appropriate number of counted particles as well as selected magnification and micrograph resolution [10].

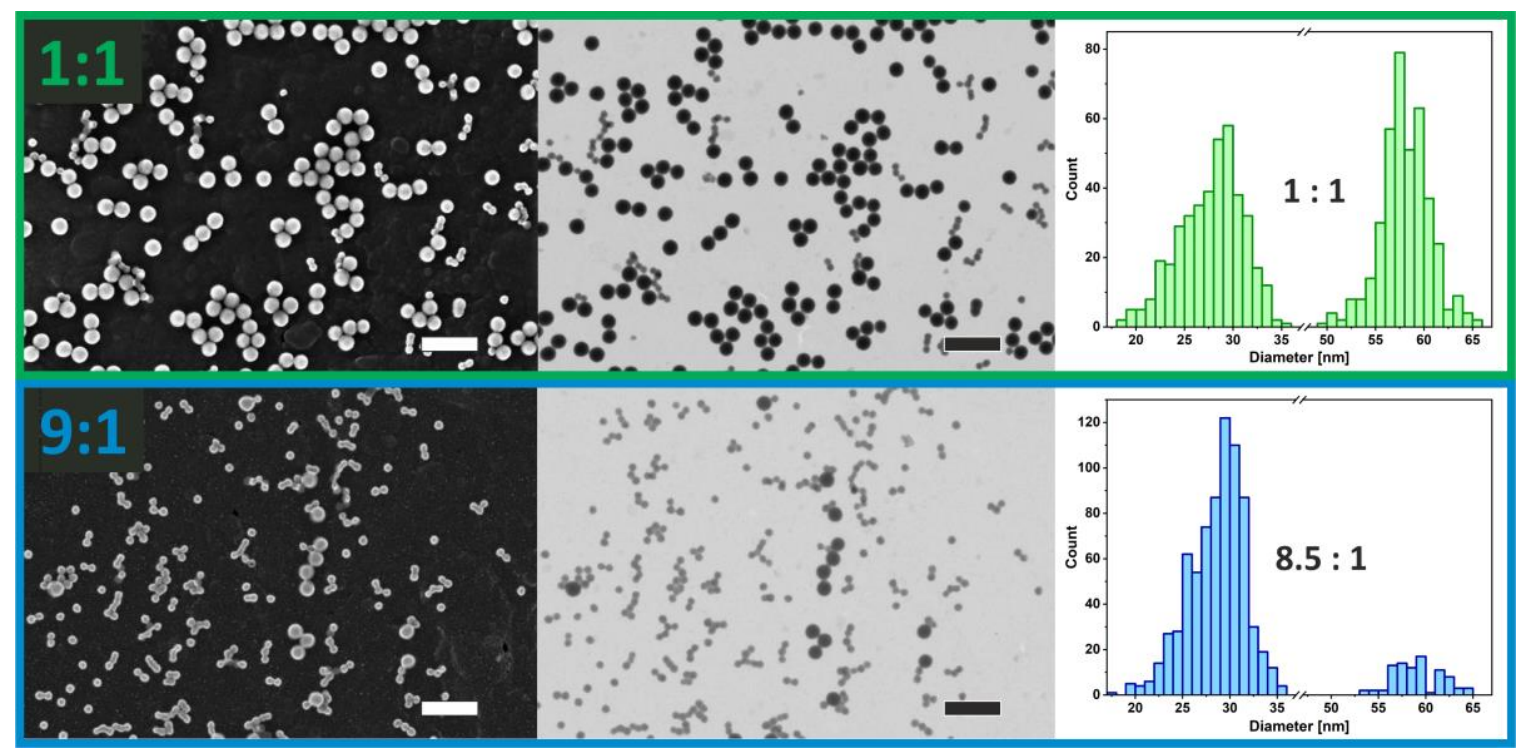

Figure 1. SEM (left), STEM-in-SEM (center) micrographs as well as respective particle size distribution (right) of the bimodal silica nanoparticles under investigation to be selected as candidate reference materials to be certified for their particle size distribution. Scale bars equal $200 \mathrm{~nm}$. Number ratios in green and blue indicate the nominal relative particle concentration while the ratio in the histograms represents the counted relative concentrations. Note that the histograms were compiled from a sequence of micrographs.

References [1] F Babick et al, J. Nanopart. Res. (2016), 18:158. [2] P-J De Temmerman et al, J. Nanopart. Res. (2014), 16:2177. [3] V-D Hodoroaba et al, Microsc. Microanal. 20 (2014), pp. 602-6012. [4] U Mansfeld et al, Microsc. Microanal. 25 (Suppl 2) (2019), p. 2328. [5] K Kumagai, A Kurokawa, Metrologia 56 (2019) 044001. [6] J Mielke et al, Microsc. Microanal. 23 (2019), pp. 163-172. [7] https://www.bam.de/Content/DE/Projekte/laufend/nPSize/npsize.html. [8] S. Fouilloux et. al, J. Colloid Interface Sci., 346 (2010), pp. 79-86. [9] S. Cuello-Nuñez et al., J. Anal. At. Spectrom. (2020), Advance article, DOI: $10.1039 / \mathrm{c} 9 \mathrm{ja} 00415 \mathrm{~g}$.

[10] This project has received funding from the EMPIR programme co-financed by the Participating States and from the European Union's Horizon 2020 research and innovation Programme. 